\title{
Review of: "Drug resistance mechanisms create targetable proteostatic vulnerabilities in Her2+ breast cancers"
}

\author{
Shirish Shenolikar ${ }^{1}$ \\ 1 Duke-NUS Medical School
}

Potential competing interests: The author(s) declared that no potential competing interests exist.

Manuscript by Singh et al used in vitro exposure of Her2+ breast cancer cells to the anti-Her2 drug, Lapatinib, as a model of acquired drug resistance in human cancers. While the general question of acquired drug resistance definitely merits detailed investigation, precisely how the authors arrived at the selected drug concentrations, namely $250 \mathrm{nM}, 500 \mathrm{nM}$ and $1 \mathrm{mM}$, or the duration of treatment that led them to define two weeks drug exposure as "resistance phase" or two months exposure as the "relapse phase" or "release of cells from lapatinib at the relapse phase" or "drug release phase - 48 hrs in drug free media" and how these somewhat arbitrary designations can be used to provide novel insights into drug resistance seen in cancer patients is entirely unclear. The subsequent omics analyses, such as RNAseqbased transcriptomic profiling (Figure 1D) or metabolomics, while expansive in their nature, are not followed up with critical validation studies. Thus, the datasets obtained from these experiments are subjected to only the most cursory analysis. Yet other experimental design flaws (listed below) compound these problems, adding up to the overall reduced impact of the findings.

\section{Specific Comments :}

1. While the experimental design outlined schematically in Figure $1 \mathrm{~A}$ suggests that cells were treated at three different drug concentrations for 24 hrs, 1 month and 2 months to promote drug resistance, some data (Figure 1D) are compared at 48hrs, 2 weeks and 2 months. While this adds some confusion, there is little evidence from the downstream Her2 signaling analyses that there was any difference seen at different drug concentrations or that with exception of 2 months, that there was any difference between 1 day and 1 month to suggest a time dependency to the acquired resistance. In addition, with exception to the omics data (Figure 1D and Figure 4A), there are at best only marginal differences between 2 months (relapse phase) and DR (drug release phase) e.g. Figures 1C, 2B, 3D, 4C and 5C...Yet the bulk of the analyses appeared to focus on the latter two conditions.

2. Figure 1B, represents an unusual measurement of "growth rate" - defined as difference in relative viability between days 1 and 3 of seeding divided by 2 . The authors could have and should have used any number of the numerous more accurate and well-accepted growth assays. Moreover, there is little description or detail provided on how the gene expression heatmaps shown in Figure 1D were analyzed 
to focus the authors' attention on NRG1 signaling (Figure 1E) other than earlier publications had indicated that HER3 receptor tyrosine kinase, which is bound by neuroregulin, was overexpressed following Her2 acquired resistance. As the folder labelled supplementary information indicated that no data were deposited, it was impossible to evaluate the supplementary figures cited in results.

3. Figure $3 A$ shows dramatic difference $p$-HER3 patterns at 1 day, 2 weeks, 2 months and DR, yet the authors elected to focus further experiments only on 2 months and DR, why? In any case, in these studies (Figures 2B and 2C) the differences between $2 \mathrm{M}$ and $\mathrm{DR}$ were minimal, why? Figure $2 \mathrm{C}$ is described in the legends as monitoring "relative viability" but labelled in the figure as "relative survival" or in Figure 2E as "relative cell growth" and in figure axis as "relative viability". These are not consistent or interchangeable terms.

4. Figure $3 A$ monitors protein synthesis using Click-It which allows for microscopic assessment of newly synthesized proteins in individual cells. These cell biological data show very clearly that there is quite significant cell to cell variation in labelling when compared with DAPI staining. How these kinds of data were transformed into the graphs shown in Figure $3 \mathrm{~B}$ and $3 \mathrm{C}$ with almost no discernable error bars is quite puzzling. In any case, another independent biochemical assay of new protein synthesis should be included to strengthen these findings.

5. Figure 3D shows data assessing the activation of ER stress with the most profound changes seen at 2 weeks rather than $2 \mathrm{M}$ or DR, where the very low P-elF2a was highlighted but many other readouts were also waning. The polyubiquitin blots are very weak and as such, may be difficult to evaluate.

6. The metabolomics studies described in Figure 4 are the weakest as none of the changes in metabolites were independently validated and the changes in glycosylated proteins (Figure 4C) provides no useful information.

7. Figure 5 is weakened by the authors use of Guanabenz as an "inhibitor of GADD34" when increasing publications (Crespillo-Casado et al (2017) ELife 6:e26109; Perego et al (2018) Sci. Signal. 11:eaam8104; Claes et al (2019) J. Biol Chem 294:13478) have argued that this drug modulates ISR by some as yet unknown mechanism. Most importantly, Guanabenz does not bind GADD34 nor impair elF2a dephosphorylation and therefore, there is reasonable doubt that it is a GADD34 inhibitor as stated in the initial studies. Thus, Figures 5B, 5D and 5F should be deleted. Instead, the authors should seriously explore the use of ISRIB (Sidrauski et al (2015) ELife 4:e05033: Rabinow et al (2019) Proc Natl Acad Sci USA 116:2097; Zyryanova et al (2021) Mol Cell 81:88) which circumvents P-elF2a to restore protein synthesis in stressed cells and thus, provides much stronger evidence for the contribution of the PERK/P-elF2a pathway than Guanabenz.

Figures $6 \mathrm{~A}, \mathrm{~B}, \mathrm{C}$ (there are two panels labelled C) and D further highlight that there was no significant difference between the $2 \mathrm{M}$ and DR samples. In any case, the significance of these data is 